\title{
BMJ Global Health Unpacking the performance of a mobile health information messaging program for mothers (MomConnect) in South Africa: evidence on program reach and messaging exposure
}

Amnesty E LeFevre, ${ }^{1,2}$ Pierre Dane,${ }^{3}$ Charles J Copley, ${ }^{4}$ Cara Pienaar, ${ }^{3}$ Annie Neo Parsons, ${ }^{3}$ Matt Engelhard, ${ }^{4}$ David Woods, ${ }^{5}$ Marcha Bekker, ${ }^{6}$ Peter Benjamin, ${ }^{7}$ Yogan Pillay, ${ }^{8}$ Peter Barron, ${ }^{8,9}$ Christopher John Seebregts, ${ }^{2,3}$ Diwakar Mohan ${ }^{1}$

To cite: LeFevre AE, Dane $P$, Copley CJ, et al. Unpacking the performance of a mobile health information messaging program for mothers (MomConnect) in South Africa: evidence on program reach and messaging exposure. BMJ Glob Health 2018;3:e000583. doi:10.1136/ bmjgh-2017-000583

- Additional material is published online only. To view, please visit the journal online (http://dx.doi.org/10.1136/ bmjgh-2017-000583).

$\mathrm{PB}, \mathrm{CJS}$ and DM are co-senior authors.

Received 21 September 2017 Revised 17 January 2018 Accepted 20 January 2018
Check for updates

For numbered affiliations see end of article.

Correspondence to Dr Amnesty E LeFevre; aelefevre@gmail.com

\section{ABSTRACT}

Despite calls to address broader evidence gaps in linking digital technologies to outcome and impact level health indicators, limited attention has been paid to measuring processes pertaining to the performance of programs. In this paper, we assess the program reach and message exposure of a mobile health information messaging program for mothers (MomConnect) in South Africa. In this descriptive study, we draw from system generated data to measure exposure to the program through registration attempts and conversions, message delivery, opt-outs and drop-outs. Using a logit model, we additionally explore determinants for early registration, opt-outs and dropouts. From August 2014 to April 2017, 1159431 women were registered to MomConnect; corresponding to half of women attending antenatal care 1 (ANC1) and nearly $60 \%$ of those attending ANC1 estimated to own a mobile phone. In 2016, $26 \%$ of registrations started to get women onto MomConnect did not succeed. If registration attempts were converted to successful registrations, coverage of ANC1 attendees would have been $74 \%$ in 2016 and $86 \%$ in 2017 . When considered as percentage of ANC1 attendees with access to a mobile phone, addressing conversion challenges bring registration coverage to an estimated $83 \%-89 \%$ in 2016 and $97 \%-100 \%$ in 2017. Among women registered, nearly $80 \%$ of expected short messaging service messages were received. While registration coverage and message delivery success rates exceed those observed for mobile messaging programs elsewhere, study findings highlight opportunities for program improvement and reinforce the need for rigorous and continuous monitoring of delivery systems.

\section{BACKGROUND}

Calls to improve the rigour and reporting of evidence on the effectiveness of digital health programs, including mobile health (mHealth), are emerging. ${ }^{1}$ mHealth programs which aim to empower women and

\section{Key questions}

What is already known?

- Mobile health (mHealth) programs which aim to empower women and catalyse demand for health services through the provision of mobile health information have been shown to increase utilisation of antenatal care (ANC), skilled birth attendance and childhood immunisation rates in a number of settings.

- Less is known however about the performance of the messaging and technology platforms underpinning these programs; affecting exposure to program content and, in turn, the summative outcome measures observed.

What are the new findings?

- From August 2014 to April 2017, 1159431 women were registered to MomConnect; corresponding to half of women attending ANC 1 (ANC1) and nearly $60 \%$ of those attending ANC1 were estimated to own a mobile phone.

- In 2016, 26\% of registrations started to get women onto MomConnect did not succeed and were deemed to have dropped-out.

- If registration attempts were converted to successful registrations, coverage of MomConnect in 2017 would have reached nearly all ANC1 attendees with access to a mobile phone.

- Among women registered, nearly $80 \%$ of expected text messages were received.

What do the new findings imply?

- Study findings highlight the need to address limitations in the current registration procedures of MomConnect, including follow-up with clients that attempt to register but fail to convert.

- Evaluations of mHealth programs must measure exposure to program content including the underlying performance of the technology platform. 
catalyse demand for health services through the provision of mobile health information have been shown to increase utilisation of antenatal care (ANC), skilled birth attendance and childhood immunisation rates in a number of settings. ${ }^{2-4}$ Less is known however about the performance of the messaging and technology platforms underpinning these programs; affecting exposure to program content and, in turn, the summative outcome measures observed. The failure to document and report on these processes and the linkages between message exposure and behaviour may hinder efforts to understand and attribute the effects observed to the program.

In Ghana, the Mobile Technology for Community Health (MOTECH)'s mobile midwife program sought to improve the uptake of reproductive, maternal, newborn and child health services by sending pregnant women and mothers of children under the age of 1 prerecorded audio health information messages timed to a women's gestational age or her infant's age. Limitations in the technological performance of the program ${ }^{5}$ meant that $<25 \%$ of mobile health information messages were received by pregnant women. ${ }^{6}$ By $6-12$ months post partum, $<6 \%$ of enrolled women were exposed to at least one message. ${ }^{6}$ These findings reinforce the need to collect evidence on summative evaluation findings, and the processes which underpin them.

The MomConnect program was established in 2014 by the South African National Department of Health (NDoH) to register pregnancies, and provide pregnant and postpartum women with twice-weekly health information text messages as well as access to a helpdesk for patient queries and feedback. ${ }^{78}$ As one of only five maternal messaging programs to exceed 1 million registered users, ${ }^{9}$ MomConnect has grown to become one of the largest mHealth programs in the world. Beyond its absolute size, little is known about the factors influencing registration, population-level coverage or exposure to MomConnect's health information content.

Expanding on efforts that describe the program, ${ }^{8}$ technology architecture ${ }^{10}$ and linkages between registration and adverse pregnancy outcomes, ${ }^{11}$ we explore evidence on the MomConnect program's reach and messaging exposure. We follow the flow of data from individual and provider mobile devices on registration attempts, through to successful registration and message delivery. We differentiate individuals who have dropped out from those who actively opt to not receive messages through a short messaging service (SMS) request, after initial registration. Finally, we explore the effects of user characteristics on the timing of registration, opt-outs and drop-outs. This analysis aims to determine whether the program performs as intended and identifies opportunities for improving the program's reach and messaging exposure.

\section{WHAT IS MOMCONNECT?}

Details on the MomConnect program are presented elsewhere. ${ }^{12} 13$ In brief, MomConnect comprises two essential components: (1) maternal health information messaging and (2) a helpdesk. In this analysis, we focus on the former. Online supplementary table 1 summarises the message delivery sets for pregnant and postpartum women, while online supplementary table 2 summarises the message delivery content by thematic area and package. Maternal messages were developed by a consortium of stakeholders led by NDoH and inclusive of global maternal health content experts, academic partners, UN agencies, technology companies and non-government organisations. Fifty topics were identified to address relevant issues related to pregnancy or the newborn infant. For each topic, an SMS message was developed within the 160 characters allowed. Messages were kept simple and understandable to lay persons emphasising inspiration and action, and/or information and action with the broader aim of encouraging the mother to play an active role in the healthcare of herself and her infant. All messages were translated into South Africa's 11 official languages and made available as an optional selection at registration.

Once finalised, maternal messages were bundled in two sets. The first set encourages pregnant women to attend facilities and receive ANC. The second focuses on essential newborn care, nutrition including infant feeding, immunisations and hygiene. Depending on the gestational age at the time of registration into MomConnect, women are eligible to receive one of three bundled message sets: (1) standard (sign-up prior to 30 weeks' gestation); (2) later (sign-up at 31-35 weeks' gestation) or (3) accelerated (sign-up at $>35$ weeks' gestation).

\section{MEASURING EXPOSURE TO MATERNAL HEALTH INFORMATION MESSAGES}

Figure 1 outlines the optimal pathway for pregnant women from point of contact with the health system, to registration into MomConnect, message delivery and receipt and the intended effects on behaviour. ${ }^{\mathrm{i}}$ In practice, multiple breaks can occur at each point along this pathway. Registration to receive MomConnect messages can be boosted through self-subscription or at community level via community health workers. Self-subscription or subscription from a community health worker at the community level triggers a short set of six messages mainly encouraging women to attend antenatal clinics. Eligibility to receive the full message set is dependent on facility-based confirmation of the pregnancy and facility-based registration via Unstructured Supplementary Service Data (USSD), ${ }^{\text {ii }}$ either on the mothers' mobile phone, on the clinic nurse's mobile phone or captured

\footnotetext{
${ }^{\text {i }}$ System generated data on careseeking and practices were not available across all provinces and thus not included in this analysis.

${ }^{i i}$ USSD is a communications protocol used by mobile phones to communicate with mobile network operator's computers. In contrast to SMS USSD messages create a real-time connection which allows for a two-way exchange of data. Common applications include the refill of balances on a user's SIM card.
} 


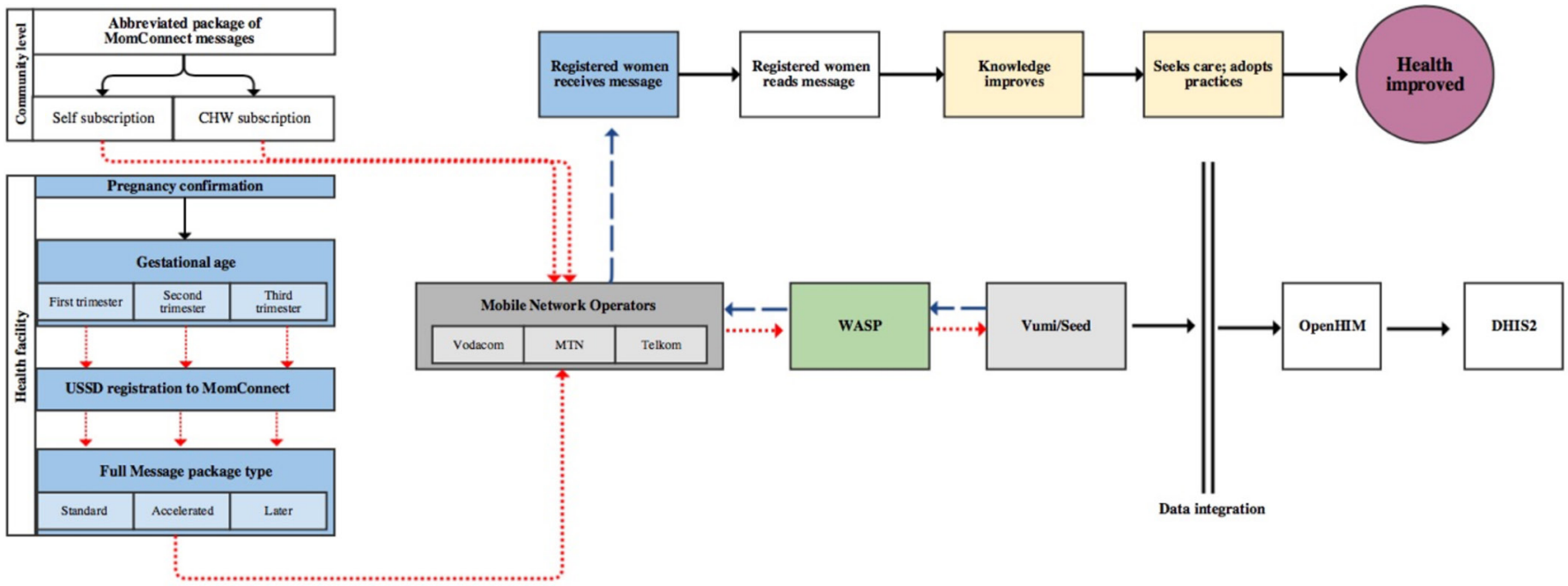

Registration flow

Message delivery flow - - -

Figure 1 Measuring the flow of registration and message delivery. The red line denotes the pathway of registration flow while the light blue line reflects the message delivery pathway. The dotted nature of the lines is intended to denote the potential for breaks in the continuity of data flow at each point in the pathway. Analyses in this paper focus only on registration and message delivery data. WASP, Wireless Access Service Provider; Vumi/SEED, Messaging engine for the delivery of SMS; OpenHIM, Middleware system for enabling interoperability with health information systems; DHIS2, District Health Information System 2, includes data on antenatal care registration.

in batches on a data clerks mobile phone. Registered women automatically receive messages. These continue until the baby is 1 year of age unless they otherwise 'optout' through SMS, either voluntarily or as a result of loss of the fetus or baby.
Table 1 summarises key definitions and measurement strategies. Exposure was measured by assessing the technological performance of the program as defined by: (1) USSD registration attempts; (2) USSD successful registrations and (3) the MomConnect platform's effectiveness

Table 1 Key terminology

\begin{tabular}{|c|c|c|}
\hline Key terms & Definition & Measurement \\
\hline Technological performance & $\begin{array}{l}\text { Aims to determine if the technology platform } \\
\text { performs as intended }\end{array}$ & $\begin{array}{l}\text { System generated data on } \\
\text { USSD registration } \\
\text { Proportion of USSD attempts at registration } \\
\text { which successfully convert to registration } \\
\text { Mean USSD tries per woman successfully } \\
\text { registered } \\
\text { Message delivery } \\
\text { Proportion of messages successfully } \\
\text { delivered }\end{array}$ \\
\hline Registration coverage & $\begin{array}{l}\text { Proportion of pregnant women who } \\
\text { successfully register to MomConnect out of all } \\
\text { women that are reported to have attended the } \\
\text { first ANC clinic in the public sector }\end{array}$ & $\begin{array}{l}\text { System generated data to yield numerator } \\
\text { data on registration; District Health Information } \\
\text { System } 2 \text { data to measure the denominator of } \\
\text { ANC1 recipients }\end{array}$ \\
\hline Opt-outs & $\begin{array}{l}\text { Registered MomConnect users who send an } \\
\text { SMS declining messages }\end{array}$ & $\begin{array}{l}\text { System generated data on } \\
\text { proportion of registered users who opt-out } \\
\text { reasons for opt-out }\end{array}$ \\
\hline Drop-outs & $\begin{array}{l}\text { Drop-outs during registration:The number of } \\
\text { unique mobile phone numbers on MomConnect } \\
\text { (msisdn) attempting to register on USSD that } \\
\text { did not convert to registration } \\
\text { Drop-outs following registration: Women } \\
\text { registered to MomConnect who fail to receive } \\
\text { messages for five consecutive weeks }\end{array}$ & $\begin{array}{l}\text { USSD registration assessed through a } \\
\text { review of system generated data }\end{array}$ \\
\hline
\end{tabular}

ANC1, antenatal care 1; SMS, short messaging service; USSD, Unstructured Supplementary Service Data. 
in 'pushing' out messages during pregnancy and post partum to registered women. Registration coverage was measured as the proportion of women successfully registered to MomConnect out of those reported in the District Health Information System 2 to have attended their first ANC clinic in a public health facility. Opt-outs are defined as registered MomConnect users who send an SMS declining future receipt of messages. Women were asked to specify one of the following reasons when opting out: miscarriage, stillbirth, baby loss, messages not useful and other. Distinct from opt-outs are registered users who 'dropped-out'. Drop-outs were assessed at two time points: (1) unique phone numbers attempting to register to MomConnect which failed to successfully convert to registration and (2) registered users who failed to receive messages for five consecutive weeks. The latter were assumed to have changed their mobile phone numbers and/or lost their devices. Among registered users, message delivery success was determined as the proportion of messages received out of those sent.

All data used in this analysis were drawn from system generated data on registration, message delivery, opt-outs, drop-outs and ANC1 utilisation. Data on overall registration trends and ANC utilisation spanned from 1 January 2015 to 30 April 2017, while USSD data were assessed from 1 January to 31 December 2016. Data on USSD attempts, registration and message delivery trends were analysed using proportions and frequencies. $\mathrm{X}^{2}$ tests were applied to assess the differences across provinces in user characteristics, including age, possession of a South Africa identification book or card (a proxy for nationality) and language. A logit model was then applied to registered users in each province to assess whether user characteristics were associated with the timing of registration, opt-outs and drop-outs. Clustering at the level of facilities was accounted for by the use of Huber White sandwich estimators. Adjusted ORs and 95\% CIs are presented.

\section{REGISTRATION INTO MOMCONNECT}

Figure 2 provides an overview of enrolment from 1 January 2016 to 31 December 2016. There were 426 631 unique phone numbers that attempted to register to MomConnect using USSD. An estimated 26\% of these $(\mathrm{n}=111$ 788) failed to convert to registration and were deemed to have dropped-out. Drop-outs during registration are attributed in part to challenges with the USSD platform which works in time-bound sessions and as well to human error in not completing the registration fields and/or dialing back in when session time-outs occur. For unique phone numbers successfully registered in 2016, a mean of 4.3 USSD sessions were required before registration was achieved. Among the $74 \%$ that did successfully register to MomConnect $(\mathrm{n}=314843), 8 \%$ were identified as provider devices and $92 \%$ were women's own mobile

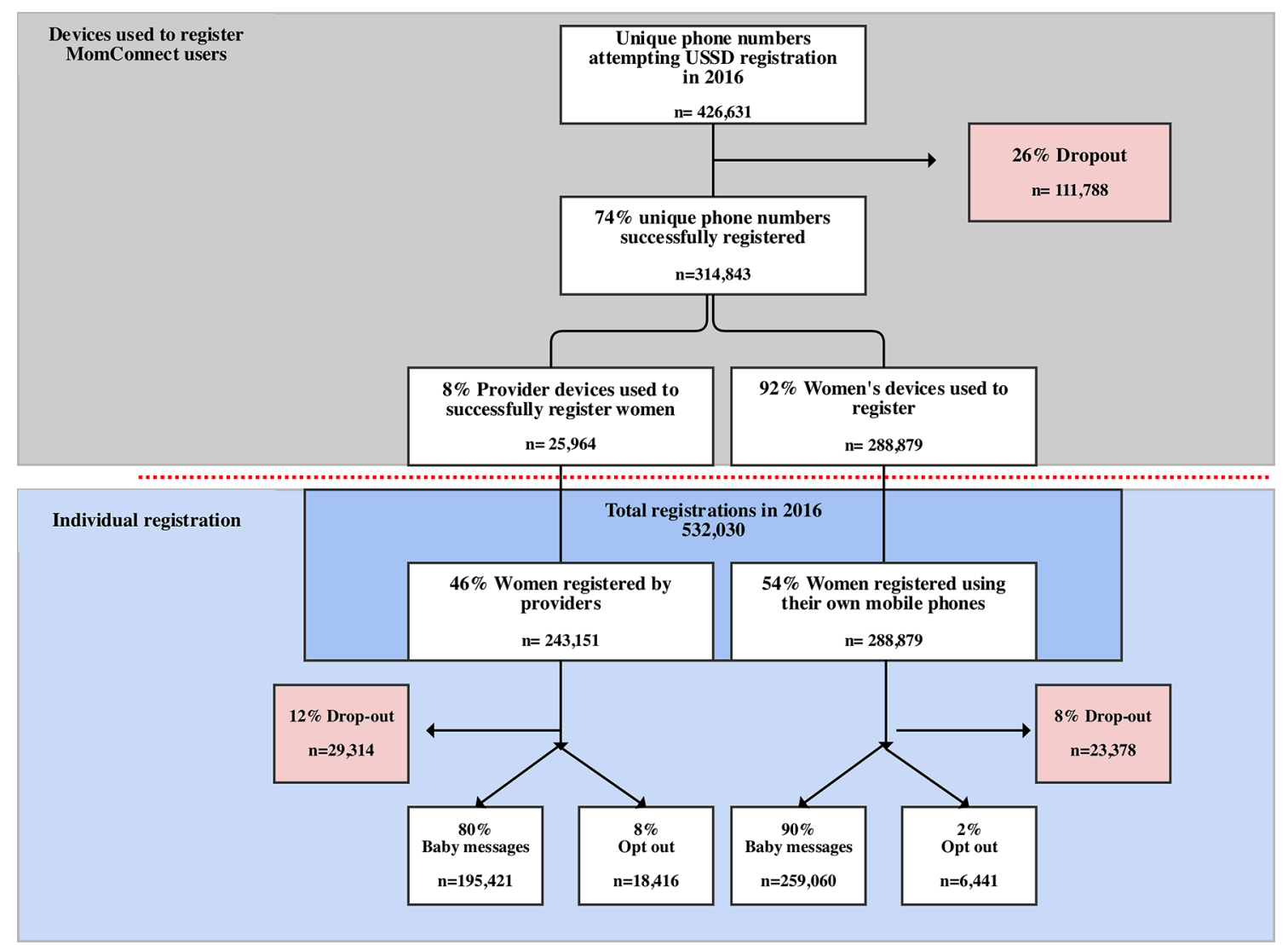

Figure 2 Overview of MomConnect enrolment flow from 1 January 2016 to 31 December 2016. USSD, Unstructured Supplementary Service Data . 
phones. ${ }^{\text {iii }}$ Collectively, this translates to 314843 devices being used to register 532030 women in $2016 .{ }^{\text {iv }}$ Provider devices $(n=25964)$ led to unique device $(m s i s d n)$ registration of 243151 women in 2016; a ratio of 9.4 registrations per device and accounted for $46 \%$ of total registrations. Over the same period, 288879 (54\%) women registered on their personal mobile device.

\section{TRANSLATING REGISTRATIONS INTO POPULATION LEVEL COVERAGE}

Table 2 presents data on the absolute number and characteristics of women successfully registered to MomConnect, while figure 3 presents trends in registration coverage overall and by province from 1 January 2015 to 30 April 2017. Nearly half of registrations came from two provinces: Gauteng (22\%) and KwaZulu-Natal (KZN, 22\%). In addition to having the lowest registration coverage as a percentage of ANC, the Northern Cape contributed $1 \%$ of MomConnect registrations (total population share $2 \%$ ).

National level trends suggest an increase over time in the proportion of women attending ANC1 registered to MomConnect from $40 \%$ in $2015,55 \%$ in 2016 , to $64 \%$ thus far in 2017. Coverage increased by a mean of $17 \%$ from 2015 to 2016 and 4\% from 2016 to mid-2017. Across provinces, registration coverage was highest in Limpopo (89\% in 2017) for all years. Slight declines in registration from 2016 to 2017 were observed in the Western Cape, North West, and Free State provinces.

We estimate that $83 \%-89 \% 1415$ of ANC1 attendees own a mobile phone, ${ }^{\mathrm{v}}$ a figure that if applied to 2016 data would translate to 805268-863481 women. Considering registration coverage as a percentage of ANC1 attendees with access to a mobile phone would shift coverage to $62 \%-66 \%$ in 2016 and $72 \%-77 \%$ in 2017. If registration attempts were converted to successful registrations, MomConnect's coverage of ANC1 attendees would have been $74 \%$ in 2016 and $86 \%$ in 2017 . When considered as percentage of ANC1 attendees with access to a mobile phone, addressing USSD conversion challenges could bring MomConnect registration coverage to $83 \%-89 \%$ in 2016 and $97 \%-100 \%$ in 2017.

In spite of USSD registration limitations, MomConnect is one of the largest maternal mobile messaging programs

\footnotetext{
iii To accommodate registration by provider devices, the USSD registration process includes an option to input a mobile number to receive messages that is different from that of the mobile device being used to register the person.

${ }^{\text {iv }}$ The MomConnect data set attempts to collect a unique identifier, that is, the South African ID Number or a passport number but this is an optional field. The mobile phone number of the device registering on MomConnect (msisdn) is obligatory and used as a proxy unique system identifier.

${ }^{v}$ While data on access to and ownership of mobile phones are limited, a 2012 report suggests that $82 \%$ of women and $86 \%$ of men reportedly owned a mobile phone in South Africa. ${ }^{15}$ A more recent report from the Pew Research Center reports that $89 \%$ of South Africans own a mobile phone; however, differentials by gender are not presented. ${ }^{14}$
}

globally in terms of absolute numbers (>500000 pregnant women in 2016) and with regard to the proportion of eligible women covered ( $>60 \%$ of all ANC1 attendees). Contextualising these data against other maternal messaging programs remains challenging because of the limited evidence available, particularly on registration coverage. The Mobile Alliance for Maternal Action (MAMA) project was one of the precursors to MomConnect in South Africa and elsewhere globally has deployments in Bangladesh, India and Nigeria. ${ }^{9}$ In South Africa, the MAMA project was implemented over a period of 3 years and led to the registration of over 500000 women. ${ }^{16}$ In Bangladesh, MAMA is delivered through the Aponjon program, which provides maternal health information messages through IVR or SMS. Since its inception 6 years ago, over 1.9 million women have been subscribed and services expanded to include a doctor's line and two additional mobile applications. ${ }^{9}$ In India, mMitra provides MAMA maternal messages using IVR to an estimated 600000 subscribers across three states. ${ }^{9}$

Elsewhere, evidence on other maternal mobile messaging programs is emerging. The Kilkari program in India provides once weekly stage-based IVR health information messages on topics ranging from family planning to maternal and child nutrition to pregnant and postpartum women up to 1-year post partum. To date, the program has been scaled across 12 states and estimates having reached a minimum of 2 million pregnant women, new mothers and their families in the last 1.5 years; corresponding to a population level coverage of pregnant women of approximately $15 \% .{ }^{17}$ In South Africa, the decision to enrol women at the facility level using mobile phones, while marred by challenges, has nevertheless meant that a larger proportion of pregnant women get covered and that nearly one-third of registrations occur within the first trimester of pregnancy thus maximising the period of exposure.

\section{WHAT ARE THE CHARACTERISTICS OF MOMCONNECT REGISTERED USERS?}

Significant differences were observed across provinces for all characteristics assessed (table 2). Findings suggest that the majority of registered users held a South African National ID card (58\%-83\%), were under 26 years of age $(29 \%-42 \%), 13-26$ weeks gestation at the time of registration $(46 \%-54 \%)$ and opted to receive messages in English (28\%-78\%). In 6 of 9 provinces, over $25 \%$ of registered users did not submit information about a South African National ID card-a factor which may indicate significant utilisation of pregnancy services by foreign nationals. While half of all users are not registered until their second trimester, longitudinal trends in the mean timing of registration by gestational age suggest first trimester registrations have increased over time.

Table 3 summarises findings from a logit model exploring determinants of early registration from 


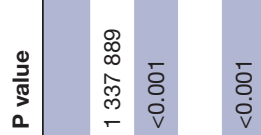

¿্ড

¿্.

¿্口

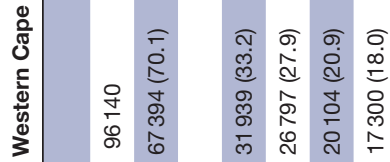

趸

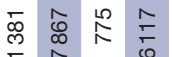

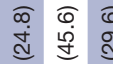

寺

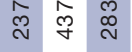

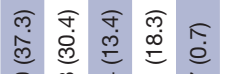

贾

为

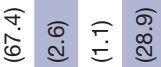

तु

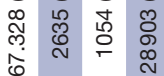

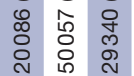

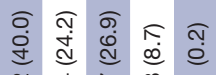

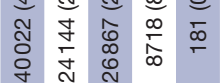

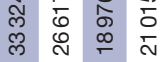

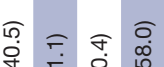

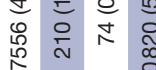

สิ

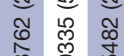

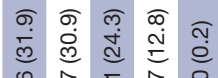

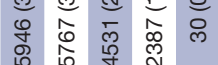

$\stackrel{\leftrightarrow}{\stackrel{\circ}{\circ}}$

$\widetilde{\infty}$

$\widehat{\widehat{\infty}}$

งิ

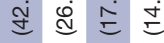

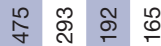

สิ

雪

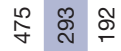

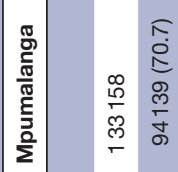

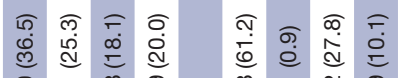

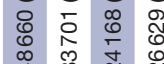

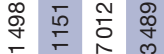

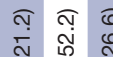

워ำ

额

ल⿸户㔾

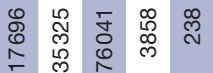

용

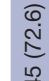

क ธุ

过过守

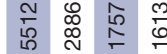

बิ

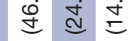

产总总魷

厄ब बิ

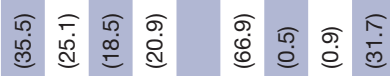

ปู่

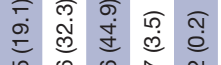

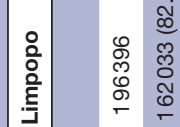

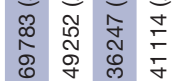

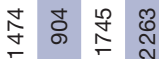

等势

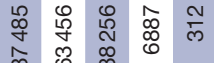

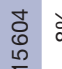

0
0
0
0

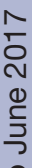

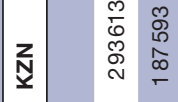

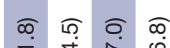

$\widetilde{\infty}=\widetilde{\infty} \widehat{\sigma}$

ब.

용

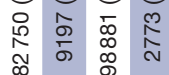

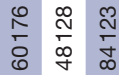

बक्ष

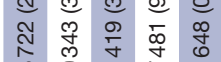

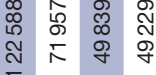

品

ก



ब约

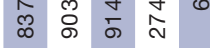

然亦

胥

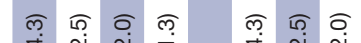

वृ

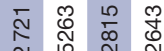

象

篎

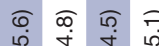

(1)

बक का एक

赵然

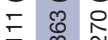

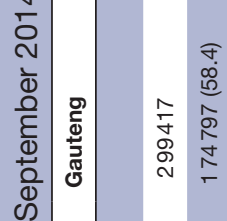

वृ

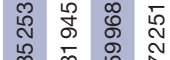

$\infty$

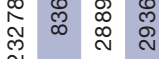

志总罂

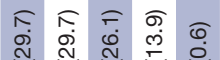

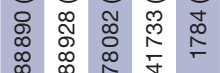

品

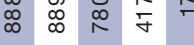

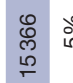

$\sqrt{2}$

$\widehat{1}$
0
0
0
0

0
0

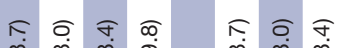

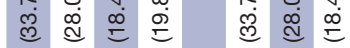

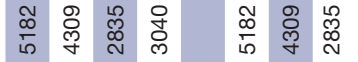

זे+

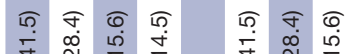

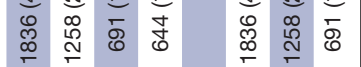

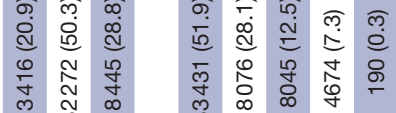

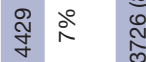

ลุ

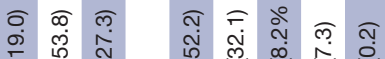

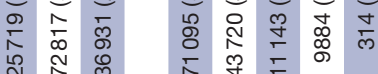

ल

守 离 $\stackrel{m}{=} \stackrel{m}{=}$ 过

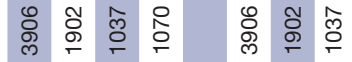

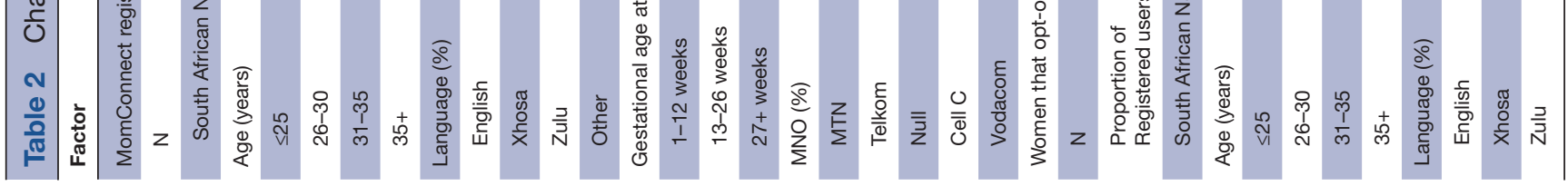


बु

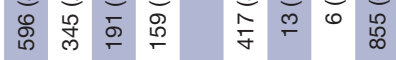

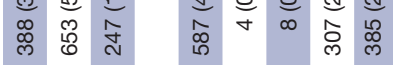
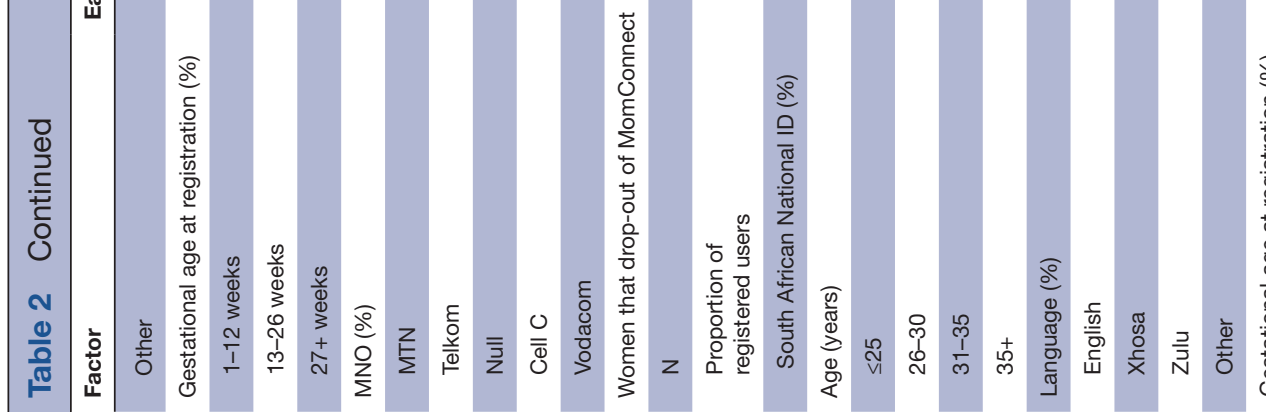

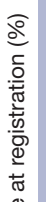




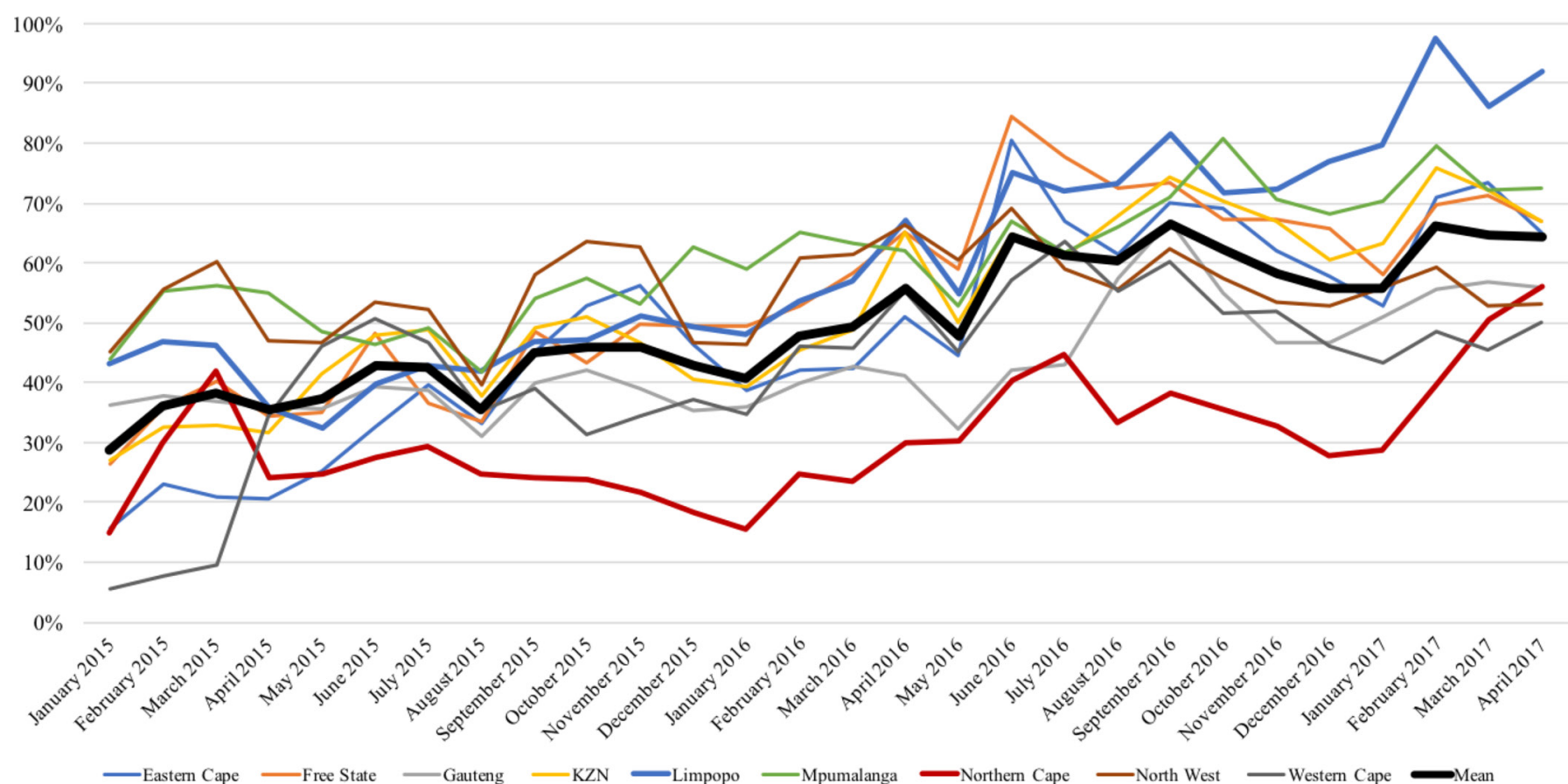

Figure 3 Percentage of MomConnect registered users out of those attending ANC1 from January 2015 to April 2017 by province.

January 2015 to April 2017. Findings suggest that the odds of early registration were significantly higher among women between 26 and 35 years of age as compared with those $<25$ years of age or higher in most provinces. Individuals not in possession of a South African ID card had a slightly higher odds of early registration in KZN, Mpumalanga and North West provinces. Language was significantly associated with early registration in all provinces except the North West and Northern Cape.

\section{DO REGISTERED USERS RECEIVE INTENDED MESSAGES?}

Figure 4 depicts trends in the proportion of messages successfully delivered by province over time, while online supplementary figure 1 presents trends in message delivery by mobile network operator (MNO). Users received over $80 \%$ of expected MomConnect messages over time. Delivery rates were stable across provinces but differed over time and by MNO type. By MNO, Telkom $(71 \%)$ and Cell C $(75 \%)$ reported the lowest message delivery success rates versus Vodacom (81\%) and MTN $(82 \%)$. Reasons for message delivery failure unfortunately were not available for all networks nor systematically assessed throughout the life of the program. However, based on the available data for 2016, the leading reason for non-delivery was that the SMS had expired-a likely indicator of an inactive phone number (online supplementary table 3 ).

'Churn'-defined by percentage of subscribers in a given time frame that cease to use mobile services for one reason or another-is an inevitable part of any program, particularly in settings such as South Africa where the majority of phones are prepaid. Given that MomConnect messages may be delivered for up to 21 months, from pregnancy to 1-year post partum, monitoring drop-outs due to churn is vital for optimising exposure to health information messages and avoiding unnecessary SMS costs. Unfortunately, MomConnect does not currently enable clients to change their registered phone number or follow-up in the event of message delivery failures. Future programs should optimally consider such measures and otherwise ensure that systems are in place to remove users in the event of repeated message delivery failures. In the case of MomConnect, in 2017 a system was put into place to classify registered users with five consecutive weeks of message delivery failure as 'inactive'. Since this system has been in place, an estimated 128839 users were discontinued-effectively saving the program costs which otherwise would have been lost in the system's efforts to push out messages to target users no longer engaged.

This line of inquiry sought to emphasise the importance of understanding exposure to the program and likely variations in message delivery as function of the technology platform, MNO coverage and user engagement over time. Overall findings on message delivery trends are challenging to contextualise given the limited reporting on this in other mHealth programs. Unfortunately, few evaluations of digital health programs have adequately explored exposure and its probable linkages with health outcomes. In Zanzibar and Malawi, programs providing mobile health information messages to expectant mothers were associated with increases in the knowledge and utilisation of services across the continuum of care. ${ }^{2}{ }^{18} 19$ While these findings are promising, the absence of details on individual level exposure to the program content greatly limits understanding of the factors influencing effects observed, their comparability with other mobile messaging programs, 


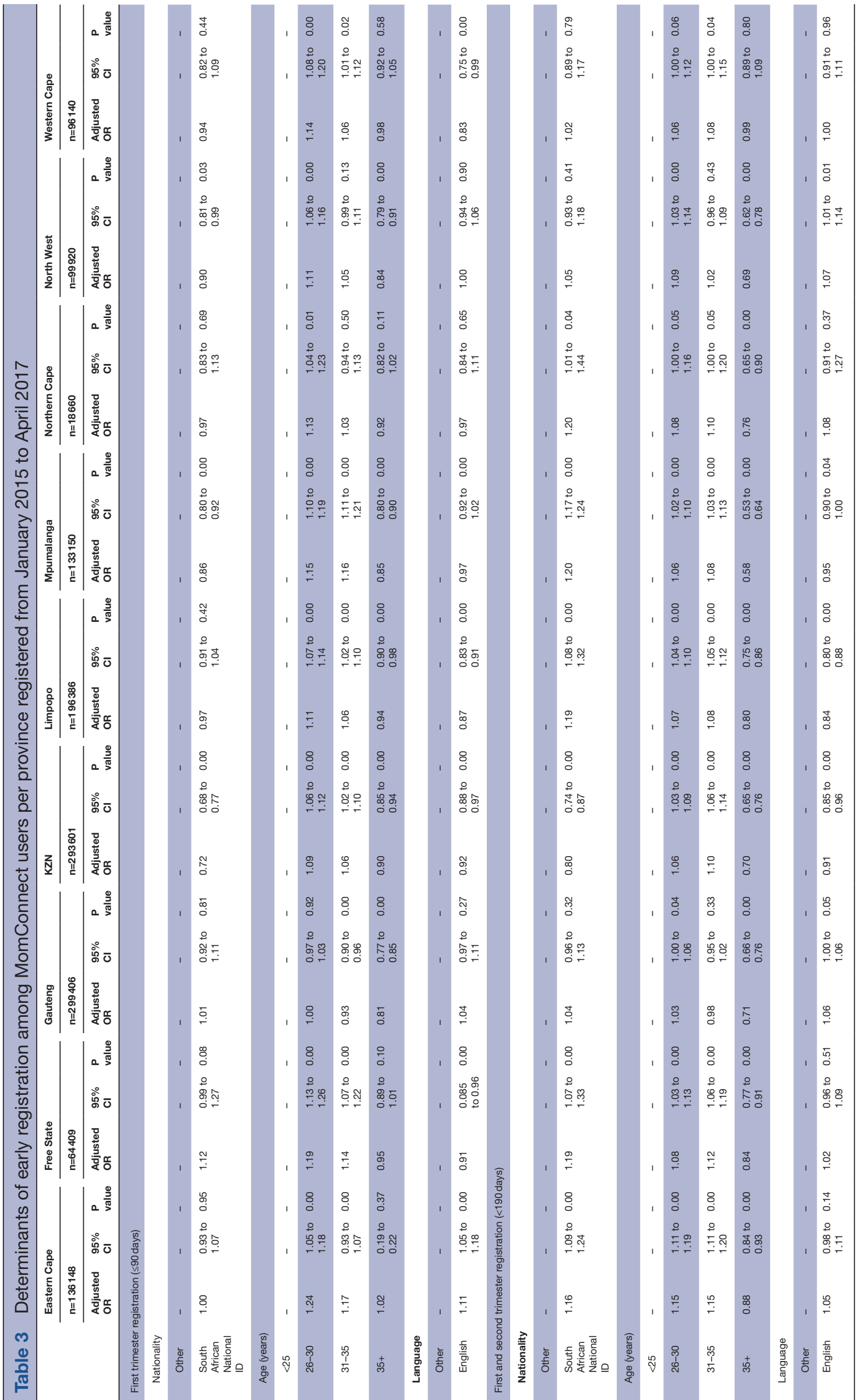




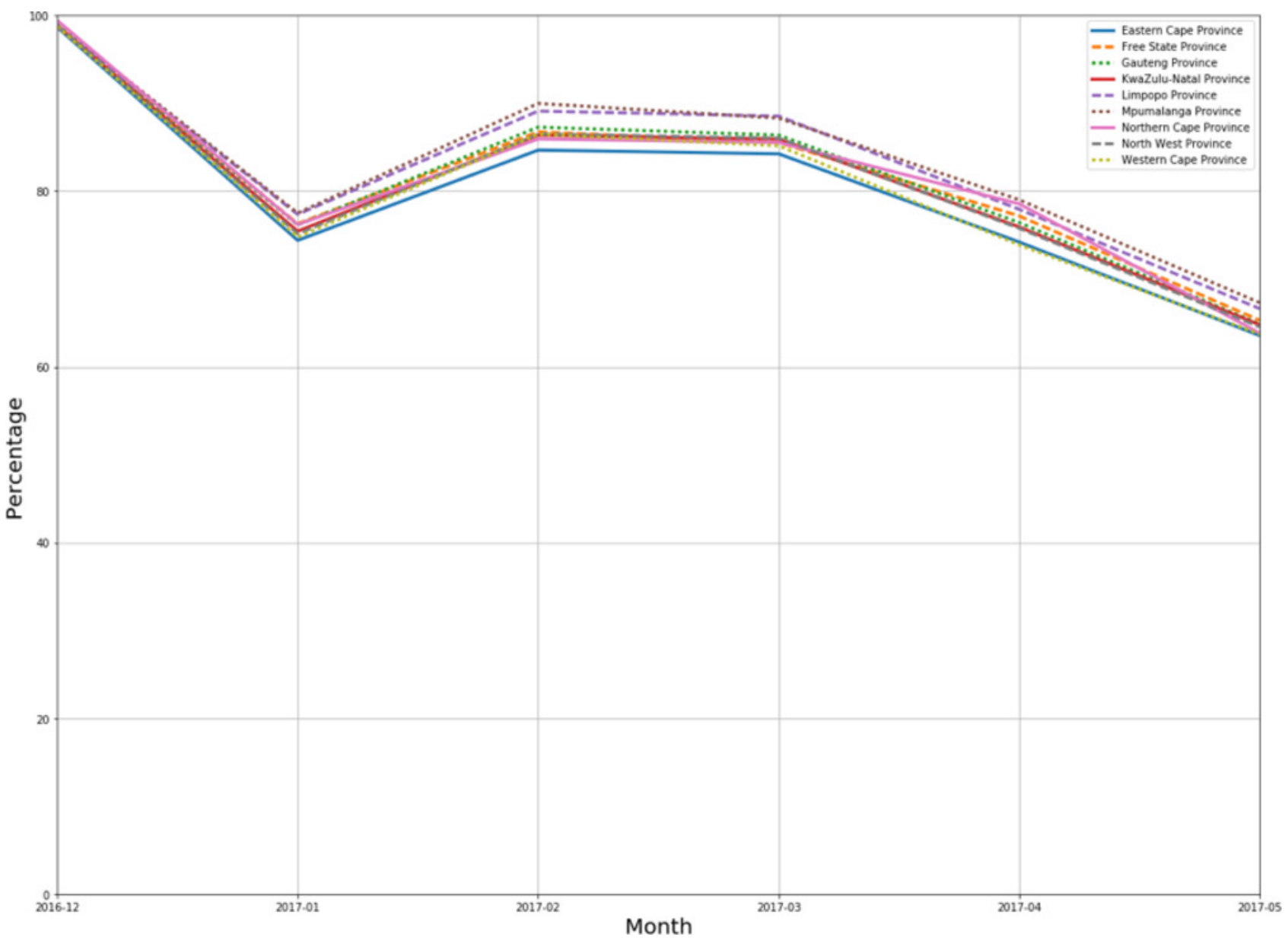

Figure 4 Message delivery success rates by month and province from December 2016 to May 2017.

and generalisability to other settings. For MOTECH in Ghana, while outcome level data were not available, analyses of IVR message delivery trends suggested that $25 \%$ or less of expected mobile health information messages were received by pregnant women, despite the majority $(>77 \%)$ owning a private mobile phone. ${ }^{6}$ For SMS programs like MomConnect, user engagement with the messages is not possible to measure through use of system generated data as the system only measures if the messages were delivered and cannot see if they were opened and/or read. However, even with slight variations by network provider, MomConnect's overall message delivery success rate was high overall, by province, $\mathrm{MNO}$ and stable with program growth.

\section{DO REGISTERED USERS STAY ENGAGED?}

Registered users were able to discontinue messaging by either 'opting-out' or 'dropping-out' (table 1). Out of the 532030 registrations in 2016, $10 \%(\mathrm{n}=52692)$ dropped out due to SMS delivery failures, $5 \%(\mathrm{n}=24857)$ opted-out and $85 \%$ went on to receive baby messages (figure 2). Individuals opting out were asked to specify the underlying reason from one of five prespecified categories (online supplementary figure 2). The leading reason for discontinuing messaging was 'other' $(63 \%)$, followed by miscarriage (12\%), stillbirth (10\%), baby loss $(7 \%)$ and messages not reportedly useful $(7 \%)$.
Characteristics associated with opt-outs and drop-outs are presented in table 2 , while table 4 presents data from a logit model exploring determinants of opt-outs and dropouts among MomConnect users. Online supplementary table 3 compares the characteristics of registered users who opt-out and drop-out of MomConnect versus those that continue to receive messages. Registered users that opted to discontinue MomConnect messages (6\%-9\%) had similar characteristics to those that dropped out. The odds of opting out were significantly higher among individuals with a South African ID card, under 25 years of age and/or who did not speak English. By comparison, drop-outs were significantly higher among non-English speakers without a South African ID and under 25 years of age.

\section{CONCLUSIONS}

Mobile maternal messaging programs hold significant promise for increasing access to critical health information and in turn bolstering knowledge, care-seeking and practices. In instances where they are nationally implemented, they too may serve as a 'gateway' for engaging users for other health services and thus, a starting point for a multitude of other supply (eg, electronic medical records, service delivery apps) and demand side (eg, alerts and reminders for care seeking) digital health solutions. 


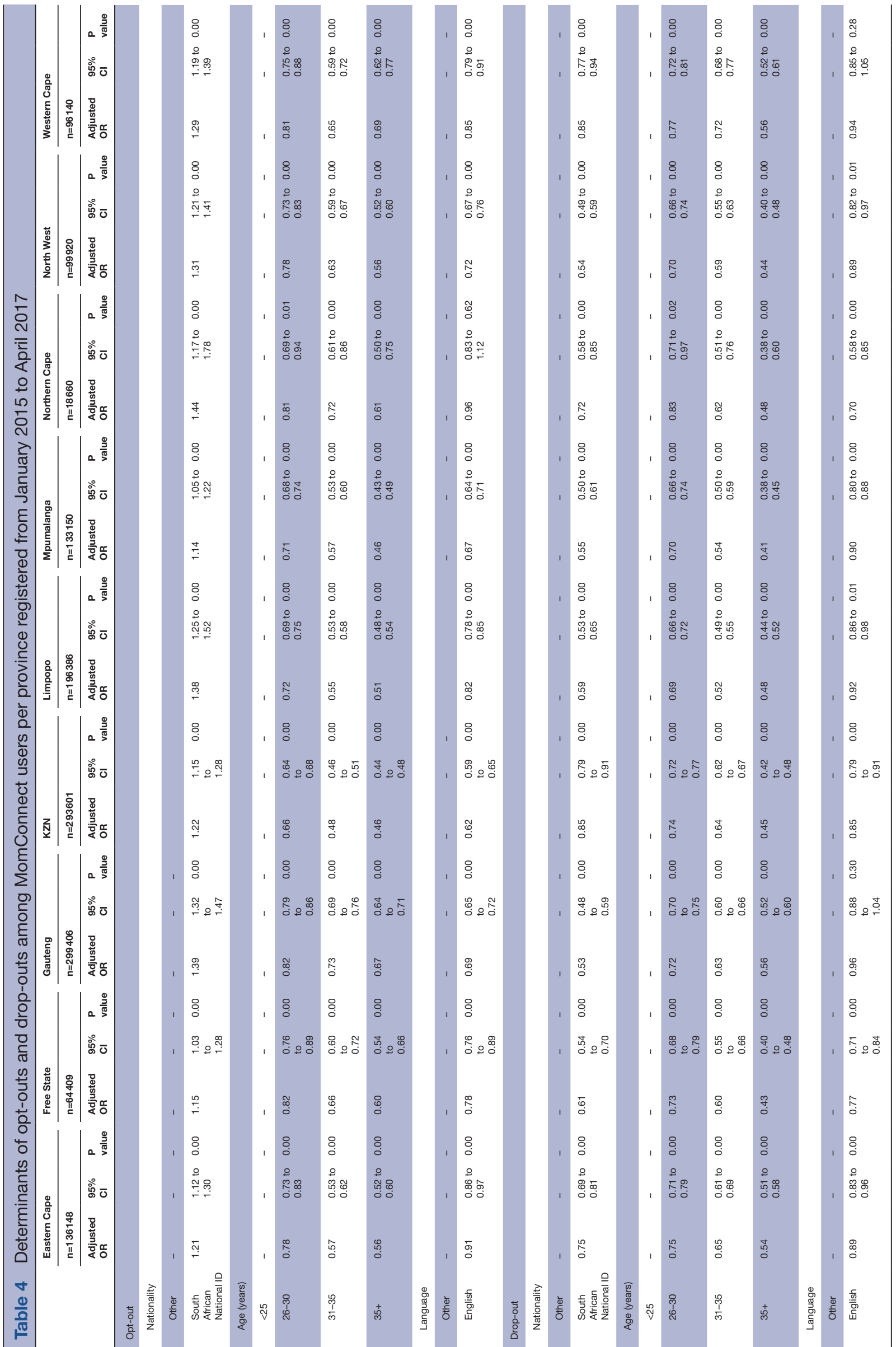


For this potential to be realised, the program must reach its intended beneficiaries and those individuals must find utility in the services received. Few evaluations of digital health solutions have sought to explore whether the program was delivered as it was intended-instead jumping over processes to measure outcome and impact level indicators without linking establishing linkages to program exposure. Study findings reinforce the need to methodically follow the flow of data to understand who receives services, in what dose and where critical breaks in the continuity of service delivery occur.

\section{Author affiliations}

${ }^{1}$ Department of International Health, Johns Hopkins Bloomberg School of Public Health, Baltimore, Maryland, USA

${ }^{2}$ School of Public Health and Family Medicine, University of Cape Town, Cape Town, South Africa

${ }^{3}$ Jembi Health Systems, Cape Town, South Africa

${ }^{4}$ Duke Department of Psychiatry \& Behavioral Sciences, Durham, North Carolina, USA

${ }^{5}$ University of Cape Town, Cape Town, South Africa

${ }^{6}$ Praekelt.org, Johannesburg, South Africa

${ }^{7}$ HealthEnabled, Cape Town, South Africa

${ }^{8}$ HIV/AIDS, TB and MCWH, National Department of Health, Pretoria, South Africa ${ }^{9}$ School of Public Health, University of the Witwatersrand, Johannesburg, South Africa

Acknowledgements The authors are grateful to Kevin Hillier of Vodacom Messaging Solutions for kindly providing registration, dropout and optout data. The support provided by John Snow, Inc. (JSI) in the President's Emergency Plan for AIDS Relief (PEPFAR) and United States Agency for International Development (USAID)-funded MEASURE Evaluation Strategic Information for South Africa (MEvalSIFSA) project to enable this publication is acknowledged with gratitude.

Contributors AEL wrote the first draft of the manuscript and conducted the analysis. PD obtained the data, conducted extensive cleaning, provided support to analyses and manuscript writing. CC obtained the data on message delivery, helped generate figures. CP, ANP and ME supported the analyses, contributed to interpretation and manuscript writing. DM, PBa and CJS oversaw the analysis and contributed to all facets of manuscript preparation. All other authors met ICMJE requirements for authorship. All authors read and approved the final manuscript.

Competing interests None declared.

Patient consent Not required.

Ethics approval National Department of Health-South Africa.

Provenance and peer review Not commissioned; externally peer reviewed.

Data sharing statement All data are available on request from the corresponding author.

Open Access This is an Open Access article distributed in accordance with the Creative Commons Attribution Non Commercial (CC BY-NC 4.0) license, which permits others to distribute, remix, adapt, build upon this work non-commercially, and license their derivative works on different terms, provided the original work is properly cited and the use is non-commercial. See: http://creativecommons.org/ licenses/by-nc/4.0/

(c) Article author(s) (or their employer(s) unless otherwise stated in the text of the article) 2018. All rights reserved. No commercial use is permitted unless otherwise expressly granted.

\section{REFERENCES}

1. Agarwal S, LeFevre AE, Lee J, et al. Guidelines for reporting of health interventions using mobile phones: mobile health (mHealth) evidence reporting and assessment (mERA) checklist. BMJ 2016;352:i1174.

2. Watterson JL, Walsh J, Madeka I. Using mHealth to Improve Usage of Antenatal Care, Postnatal Care, and Immunization: A Systematic Review of the Literature. Biomed Res Int 2015;2015:1-9.

3. Free C, Phillips G, Watson L, et al. The effectiveness of mobilehealth technologies to improve health care service delivery processes: a systematic review and meta-analysis. PLoS Med 2013:10:e1001363.

4. Free C, Phillips G, Galli L, et al. The effectiveness of mobile-health technology-based health behaviour change or disease management interventions for health care consumers: a systematic review. PLoS Med 2013;10:e1001362.

5. MacLeod BB, Phillips J, Stone A, et a/The Architecture of a Software System for Supporting Community-based Primary Health Care with Mobile Technology: The Mobile Technology for Community Health $(\mathrm{MoTeCH})$ Initiative in Ghana. Online J Public Health Inform 2012;4:1-17.

6. LeFevre AE, Mohan D, Hutchful D, et al. Mobile Technology for Community Health in Ghana: what happens when technical functionality threatens the effectiveness of digital health programs? BMC Med Inform Decis Mak 2017;17:27.

7. Peter JE, Barron P, Pillay Y. Using mobile technology to improve maternal, child and youth health and treatment of HIV patients. $S$ Afr Med J 2015;106:3-4

8. Barron P, Peter J, LeFevre AE, et al. Mobile health messaging service and helpdesk for South African mothers (MomConnect): history, successes and challenges. BMJ Glob Health 2018;3(Supp 2): 0000559

9. JHPIEGO. Lessons from Country Programs Implementing the Mobile Alliance for Maternal Action (MAMA) Program in Bangladesh, South Africa, India and Nigeria, 2010 - 2016. Baltimore, MD USA JHPIEGO, 2017.

10. Seebregts C, DaneP, Parsons AN, et al. Designing for scale: optimising the health information system architecture for mobile maternal health messaging in South Africa (MomConnect). BMJ Glob Health 2018;3(Suppl 2):e000563.

11. Heekes A, Tiffin N, Dane P, et al. Self-enrolment antenatal health promotion data as an adjunct to maternal clinical information systems in the Western Cape Province of South Africa. BMJ Glob Health 2018;3(Suppl 2):e000565.

12. Barron $P$, Pillay $Y$, Fernandes $A$, et al. The MomConnect mHealth initiative in South Africa: Early impact on the supply side of $\mathrm{MCH}$ services. J Public Health Policy 2016;37(Suppl 2):201-12.

13. Seebregts C, Barron P, Tanna G, et al. MomConnect: an exemplar implementation of the Health Normative Standards Framework in South Africa. South African Health Review Health Systems Trust 2016:125-35

14. Deen-Swarray M, Gillwald A, Morrell A, et al. Lifting the veil on ICT gender indicators in Africa. Cape Town: University of Cape Town, 2012.

15. Pew Research Center. Global Digital Communication: Texting, Social Networking Popular Worldwide. Washington, DC: Pew Research Center, 2012.

16. Coleman J, Bohlin KC, Thorson A, et al. Effectiveness of an SMSbased maternal $\mathrm{mHealth}$ intervention to improve clinical outcomes of HIV-positive pregnant women. AIDS Care 2017;29:890-7.

17. Chamberlain S. Person communication on the status of maternal mobile messaging in India. India: Kilkari, 2017.

18. Lund S, Nielsen BB, Hemed M, et al. Mobile phones improve antenatal care attendance in Zanzibar: a cluster randomized controlled trial. BMC Pregnancy Childbirth 2014;14:29.

19. Robinson A, Dalious M. Evaluation of the Information and Communications Technology for Maternal, Newborn and Child Health Project" Invest in Knowledge Initiative. Malawi: Village Reach, 2014. 